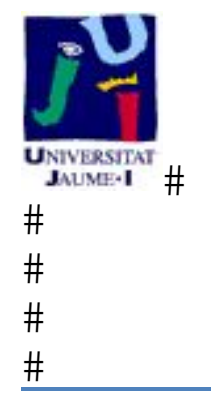

Título artículo / Títol article:

Polytopic invariant and contractive sets for closed-loop discrete fuzzy systems

$\begin{array}{ll}\text { Autores / Autors } & \begin{array}{l}\text { Ariño Latorre, Carlos Vicente ; Pérez Soler, } \\ \text { Emilio ; Sala, Antonio ; Bedate Boluda, Fernando }\end{array} \\ & \text { Journal of the Franklin Institute }\end{array}$

Versión / Versió: $\quad$ Post-print

Cita bibliográfica / Cita

ARIÑO, Carlos, et al. Polytopic invariant and bibliogràfica (ISO 690): contractive sets for closed-loop discrete fuzzy systems. Journal of the Franklin Institute, 2014, vol. 351, no 7, p. 3559-3576

url Repositori UJI:

http://hdl.handle.net/10234/125204

\# 


\title{
Polytopic Invariant and Contractive Sets for Closed-Loop Discrete Fuzzy Systems
}

\author{
Carlos Ariño ${ }^{a, *}$, Emilio Pérez ${ }^{\mathrm{a}}$, Antonio Sala ${ }^{\mathrm{b}}$, Fernando Bedate $^{\mathrm{a}}$ \\ ${ }^{a}$ Departamento de Ingeniería de Sistemas Industriales y Diseño, Universitat Jaume I, Avenida Vicent Sos Baynat, $s / n$. \\ 12071 Castelló de la Plana, Spain. \\ ${ }^{b}$ Instituto Universitario de Automática e Informática Industrial. Universidad Politécnica de Valencia. Camino de Vera S/N \\ 46022 - Valencia, Spain.
}

\begin{abstract}
In this work a procedure for obtaining polytopic $\lambda$-contractive sets for Takagi-Sugeno fuzzy systems is presented, adapting well-known algorithms from literature on discrete-time linear difference inclusions (LDI) to multi-dimensional summations. As a complexity parameter increases, these sets tend to the maximal invariant set of the system when no information on the shape of the membership functions is available. $\lambda$-contractive sets are naturally associated to level sets of polyhedral Lyapunov functions proving a decay-rate of $\lambda$. The paper proves that the proposed algorithm obtains better results than a class of Lyapunov methods for the same complexity degree: if such a Lyapunov function exists, the proposed algorithm converges in a finite number of steps and proves a larger $\lambda$-contractive set.
\end{abstract}

(C) 2013 Published by Elsevier Ltd.

Keywords: Invariant sets, Takagi-Sugeno Fuzzy Models, Contractive sets

\section{Introduction}

A large class of nonlinear systems can be exactly expressed, locally in a compact region of interest (denoted as $\Omega$ in the sequel), as a fuzzy Takagi-Sugeno (TS) model, using the "sector nonlinearity" methodology [1], [2] expressing the nonlinearity as a convex time-varying combination of "vertex" linear equations.

Once these locally exact fuzzy models are available, stability and control design for such systems can be handled in some cases via widely-used Linear Matrix Inequality (LMI) results in literature $[3,4,5,6,7]$. Most of the results can be applied to, for instance, finding a Lyapunov function $V(x)$ such that $V\left(x_{k+1}\right)<V\left(\lambda x_{k}\right)$, for some $\lambda<1$ related to geometric decay-rate [8]. Hence, the largest level set of $V$ in region of interest $\Omega$, let's denote it as $T$, is $\lambda$-contractive [9] in the sense that $x_{k} \in T \Rightarrow x_{k+1} \in \lambda T$ where $\lambda T$ denotes the linear scaling of the original set $T$ for some "contraction rate" $\lambda$. All $\lambda$-contractive sets for $\lambda<1$ are subsets of the domain of attraction of the origin, with a guaranteed decay rate.

In TS-LMI literature, the stability problem is usually considered solved once a feasible Lyapunov function $V(x)$ is found. It is usually in the form $V(x)=x^{T} P x$, or a convex combination of quadratics (fuzzy Lyapunov function [10, 1]). However, the largest level set $\left\{V(x)<V_{c}\right\}$ in the region of interest $\Omega$ may be a small subset of it, and the actual domain of attraction of the origin may be much larger. Also $V(x)$ may be non-unique.

*arino@uji.es (Carlos Ariño) 
The above issue is the motivation for the present work: trying to obtain the largest $\lambda$-contractive set for some a priori fixed $\lambda$, departing from the standard Lyapunov approach.

A computationally viable invariant and contractive-set approach to stability analysis and control originated in the 1990s (see $[9,11]$ ). It was further developed from thoughts in predictive-control literature, where invariant sets are a key ingredient for stability guarantees [12]. The MPT toolbox [13] is a widely-used piece of software to solve invariant-set and explicit predictive control problems. However, to the authors' knowledge, an application of such ideas in the nonlinear fuzzy control setup has not been yet developed. Indeed, invariant set computations for nonlinear systems is a challenging task and some assumptions and simplifications must be considered, as later discussed.

The seminal work [9] sets up most relevant issues in the relationship between stability and contractive sets.

The main idea in the above-cited approach is finding the set of states which, after $N$ steps $(N$ is an arbitrary integer) have not left the region of interest $\Omega$ (actually, the scaled $\lambda^{N} \Omega$ if $\lambda$-contractiveness is considered). If the set after $N$ steps is identical to that after $N+1$ steps, then a maximal $\lambda$-contractive set $(N \rightarrow \infty)$ has been obtained [9, 14]. A tractable algorithm following the described approach was first introduced for linear systems with linear constraints in [15]. Later, modifications have been proposed to cope with systems with polytopic uncertainties [14, 16], bounded additive disturbances [17] and switched linear systems (as a general case of piecewise-affine-systems) [18]. For all these cases the maximal invariant set was shown to be polytopic, thus, the set is a level set of a polyhedral Lyapunov function.

The basic difference between TS-fuzzy literature and robust-linear one is the fact that in nonlinear fuzzy control the convex combination coefficients are assumed known (denoted as membership functions); hence, better performance can be extracted (at least theoretically [19]) from "fuzzy" controllers (partial distributed compensators PDC, etc.) than from "robust" ones (such as those in [9]). However, the resulting closedloop expressions are polynomial in the membership functions, whereas in polytopic-uncertain systems under linear robust control such expressions are linear in the memberships. Of course, naively embedding the polynomial expressions in linear ones (blossoming) results in conservative models.

The objective of this paper is presenting a methodology to obtain the "largest" $\lambda$-contractive set in the modelling region $\Omega$, for a given $\lambda$, for discrete-time PDC closed-loop TS systems (up to conservatism in fuzzy summations due to shape-independence [19]). The domain of attraction estimates are proved larger than those obtained with a wide class of Lyapunov level-set LMI results in literature. Furthermore, this paper proves that the maximal shape-independent (i.e., disregarding the fact that the membership functions in TS systems are actually a function of the state) $\lambda$-contractive set is asymptotically obtained as the degree of complexity of a Polya multiple summation tends to infinity, i.e., the algorithm is asymptotically exact.

The structure of the paper is as follows: Section 2 discusses preliminary definitions and states the goal of the paper. Section 3 introduces shape-independent one-step sets for fuzzy control systems, together with a procedure to obtain polytopic subsets which can be proved to be asymptotically exact. These sets are used in Section 4 to propose an algorithm for the computation of polytopic invariant/ $\lambda$-contractive sets. Section 5 shows the relation of the polytopic sets obtained in this proposal with those arising from polytopic linear difference inclusions (LDI). Section 6 proves that these polytopic sets contain a certain class of ellipsoidal invariant $/ \lambda$-contractive sets derived from Lyapunov LMI results; conditions for algorithm convergence are also given. Finally, some examples appear in Section 7, and a conclusion section closes the paper.

\section{Preliminaries and problem statement}

Consider a discrete-time nonlinear system

$$
x_{k+1}=f\left(x_{k}, u_{k}\right)
$$

This system can be expressed locally in a compact region of the state-space, denoted as region of interest $\Omega$, as a TS fuzzy system with $r$ rules or local models in the form:

$$
x_{k+1}=\sum_{i=1}^{r} \mu_{i}\left(x_{k}\right)\left(A_{i} x_{k}+B_{i} u_{k}\right)
$$


where $x_{k} \in \mathbb{R}^{n}$ represents the state vector and $u_{k} \in \mathbb{R}^{m}$ the control actions, and $\mu_{i}(x)$ represents membership functions such that the vector of membership functions $\mu(x)$ belongs to the $(r-1)$-dimensional standard simplex $\Delta \subset \mathbb{R}^{r}$, defined as:

$$
\Delta=\left\{\mu_{i} \in \mathbb{R} \mid \sum_{i=1}^{r} \mu_{i}=1, \quad \mu_{i} \geq 0 \quad i: 1 \ldots r\right\}
$$

Considering now system (1) under a control law $u=h\left(x_{k}\right)$, the closed-loop equations are $x_{k+1}=f\left(x_{k}, h\left(x_{k}\right)\right)$. If a fuzzy PDC state-feedback controller [3] is used,

$$
u_{k}=-\sum_{i=1}^{r} \mu_{i}\left(x_{k}\right) F_{i} x_{k}
$$

the closed loop has the 2-dimensional summation form:

$$
x_{k+1}=\sum_{i=1}^{r} \sum_{j=1}^{r} \mu_{i}\left(x_{k}\right) \mu_{j}\left(x_{k}\right)\left(A_{i}-B_{i} F_{j}\right) x_{k}
$$

i.e., it is an homogeneous polynomial in $\mu_{i}(x)$ of degree 2 .

In the following, the region of interest $\Omega$ will be considered to be a polytope. If so wished, it may be considered as the intersection of the region in which the sector-nonlinearity model has been obtained and those in which some constraints on state and input hold (for instance, to avoid saturation). When these constraints are affine the set in which state and input should be forced to lie can be expressed as:

$$
\Lambda=\left\{(x, u) \in \mathbb{R}^{n+m} \mid T x+S u+p \leq 0\right\}
$$

where $T, S$ and $p$ are matrices with the appropriate size.

Under the control law (4), the set $\Omega$ depends on the membership functions $\mu_{i}(x)$ and it is, in general, non-polytopic:

$$
\Omega=\left\{x \in \mathbb{R}^{n} \mid \sum_{i=1}^{r} \mu_{i}(x)\left(T-S F_{i}\right) x+p \leq 0\right\}
$$

This set is characterised by a collection of possibly complicated nonlinear inequalities as $\mu_{i}$ are nonlinear functions of $x$. However, due to the fact that all membership functions are positive, a polytopic shapeindependent subset $\Omega^{[s i]} \subset \Omega$ can be defined as

$$
\Omega^{[s i]}=\left\{x \in \mathbb{R}^{n} \mid\left(T-S F_{i}\right) x+p \leq 0 \forall i=1 \ldots r\right\}
$$

disregarding the dependence of $\mu_{i}$ in $x$ and considering them to be arbitrary time-varying signals. By vertical juxtaposition of each $T-S F_{i}$ and $p$, the above set can be expressed as:

$$
\Omega^{[s i]}=\left\{x \in \mathbb{R}^{n} \mid R x+s \leq 0\right\}
$$

for some matrices $R$ and vector $s$. To avoid superscript ${ }^{[s i]}$ symbols everywhere, on the following, all references to the region of interest $\Omega$ will be assumed to actually refer to the shape-independent subset $\Omega^{[s i]}$.

\subsection{Invariant and $\lambda$-contractive sets for general nonlinear systems}

In order to properly state the problem, some preliminary definitions derived from [14] are needed.

Definition 1 Given an arbitrary target set $\mathbb{T} \subset \Omega$, the closed loop one-step set $Q(\mathbb{T})$ is the set of states in $\mathbb{R}^{n}$ from which the next state of the closed loop system $x_{k+1}=f\left(x_{k}, h\left(x_{k}\right)\right)$ is guaranteed to belong to $\mathbb{T}$, i.e.,

$$
Q(\mathbb{T})=\left\{x_{k} \in \mathbb{R}^{n} \mid f\left(x_{k}, h\left(x_{k}\right)\right) \in \mathbb{T}\right\}
$$

Consider now Algorithm 1 from [15], recursively generating a sequence of sets $\mathbb{K}_{i}(\Omega), i=0, \ldots, N$. 


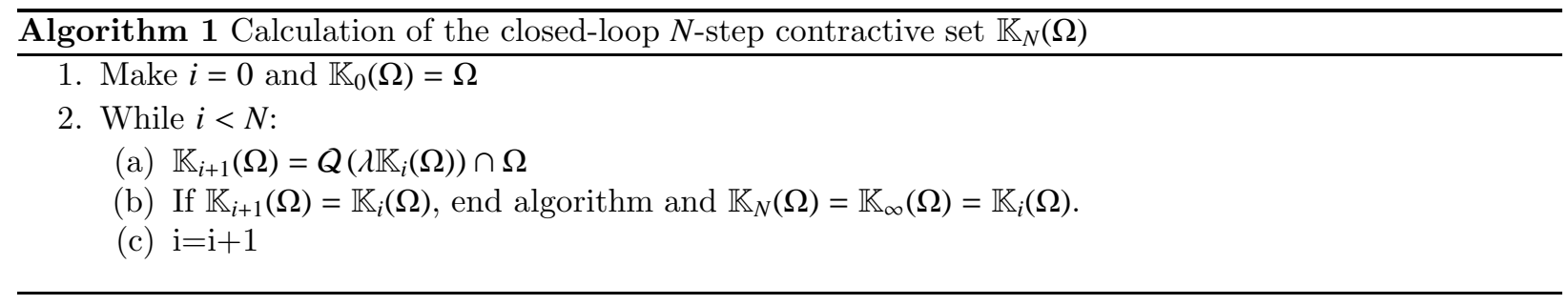

Definition 2 (Maximal $\lambda$-contractive Set) The maximal closed-loop $\lambda$-contractives set under the control law $u=h(x)$, denoted as $O_{\infty}(\Omega)$, is defined to be the set $\mathbb{K}_{\infty}(\Omega)$.

Note that a $\lambda$-contractive set with $\lambda=1$ is an invariant set.

The above set is the set of initial states for which the closed-loop trajectory never leaves the region of interest $\Omega$. This is of particular interest in the case of TS fuzzy systems as leaving $\Omega$ would invalidate the model, motivating this paper.

Relation to Lyapunov functions. Obviuosly, $\lambda$-contractive sets and Lyapunov level sets are related: if a Lyapunov function $V(x) \geq 0$ verifies $V\left(x_{k+1}\right)<V\left(\lambda x_{k}\right), V(\lambda x) \leq V(x)$, then its level sets are $\lambda$-contractive. Hence, by definition, the maximal $\lambda$-contractive set $O_{\infty}(\Omega)$ obtained with the generic (nonlinear) algorithm 1 will always succeed in finding a domain of attraction of the origin greater or equal than those resulting from any conceivable Lyapunov function.

As TS systems always admit a convex Lyapunov function (see [20] and later sections of this work), there is no loss of generality in requiring $V(\lambda x) \leq V(x)$ in the shape-independent TS setup in this paper ${ }^{1}$.

\subsection{Problem statement}

Algorithm 1 is based in the recursive computation of the one-step set, $Q(\Omega)$. In a general case, the required computations in the above Algorithm may be impossible, as the shapes of the sets will be very complex, arising from the nonlinearities in $f$ and $h$ (which, after the equivalent TS modelling are actually translated to corresponding nonlinearities in $\mu_{i}(x)$ ). As such, exact computations are only found in the literature for a limited class of systems: linear [15], polytopic [14, 16], systems with additive disturbances [17], switched linear systems [18] and linear systems with non-convex constraints [21].

Note, however, that the closed-loop fuzzy systems are in general not polytopic but polynomially dependent on the membership functions. Hence, the purpose of this paper is extending the above ideas to discrete-time systems characterised by homogeneous multi-dimensional fuzzy summations [22]. Of course, for the case of fuzzy systems, $Q(\Omega)$ is also hard to compute due to the nonlinearity of the membership functions.

The goal of this paper is to obtain the "largest possible" shape-independent polytopic subset of $Q(\Omega)$, and, subsequently, of the "largest possible" shape-independent polytopic subset of $\mathbb{K}_{N}(\Omega, \Omega)$ (possibly nonpolytopic) by adapting the above algorithms.

The accuracy of the results will depend on the relaxations of fuzzy summations to be used, which, however, are asymptotically exact [22].

Although developments are presented for double fuzzy summations arising from usual closed-loop expressions, they are easily generalizable to higher fuzzy summation dimensions (details omitted for brevity).

In this way, the obtained results in this paper will be a subset of the maximal invariant set of the original nonlinear system (1), as system (1) corresponds to a particular choice $\mu(x)$; this source of conservativeness in fuzzy systems analysis is well known [5, 23].

\footnotetext{
${ }^{1}$ This inequality requires the line from the origin to a point $x$ to be included in the Lyapunov level set associated to $x$, i.e., it must be a "starred" set. This may be conservative in general nonlinear systems but not in "linear in the state" ones, such as the TS class.
} 


\section{Shape-Independent one-step sets for fuzzy control systems}

As discussed above, Algorithm 1 is based in the recursive computation of the one-step input admissible set, $Q(\Omega)$. In the fuzzy context, the goal of computing the "true" $Q(\Omega)$ will be (conservatively) transformed to obtaining a suitable polytopic subset of $Q(\Omega)$, in fact of $Q\left(\Omega^{[s i]}\right)$ as previously discussed.

Computing invariant sets for the case with a linear controller $h(x)=-F x$ for a polytopic-uncertain system is well known in literature [16].

In this paper we generalise the argumentations to multi-dimensional convex summations, as follows.

Let us consider system (2) with a PDC fuzzy controller (4). Then, the closed-loop system can be represented as (5).

In this case, from (9), the set leading to the shape-independent $\Omega$ in one step is:

$$
Q(\Omega)=\left\{x \in \mathbb{R}^{n} \mid \sum_{i=1}^{r} \sum_{j=1}^{r} \mu_{i}(x) \mu_{j}(x) R\left(A_{i}-B_{i} F_{j}\right) x+s \leq 0\right\}
$$

We can multiply vector $s$ by $\sum_{i, j} \mu_{i}(x) \mu_{j}(x)=1$ and the one step set can be expressed as

$$
Q(\Omega)=\left\{x \in \mathbb{R}^{n} \mid \sum_{i=1}^{r} \sum_{j=1}^{r} \mu_{i}(x) \mu_{j}(x) Q_{i j}(x) \leq 0\right\}
$$

where the vector $Q_{i j}(x)$ is defined as

$$
Q_{i j}(x)=R\left(A_{i}-B_{i} F_{j}\right) x+s
$$

The set $Q(\Omega)$ is in general non polytopic as $\mu_{i}$ are nonlinear functions of $x$. Hence, for the computation of the one-step sets in Algorithm 1 a subset, $Q^{[s i]}(\Omega)$, is defined by considering $\mu_{i}$ to be arbitrary scalars ranging over the whole simplex $\Delta$, removing its relationship with $x$.

Then, the membership-shape-independent one-step set will be given rewriting (11) as:

$$
Q^{[s i]}(\Omega)=\left\{x \in \mathbb{R}^{n} \mid \sum_{i=1}^{r} \sum_{j=1}^{r} \mu_{i} \mu_{j} Q_{i j}(x) \leq 0 \forall \mu \in \Delta\right\}
$$

Evidently, $Q^{[s i]}(\Omega) \subset Q(\Omega)$ because $Q(\Omega)$ considers a particular "shape" $\mu_{i}(x)$ instead of all possible ones.

If the shape-independent set $Q^{[s i]}(\Omega)$ were used in Algorithm 1, the algorithm would obtain the largest "shape-independent $\lambda$-contractive set" $O_{\infty}^{[s i]}(\Omega)$ guaranteed to be robustly invariant for all possible pairs of model and its associated PDC controller.

\subsection{Computable subset of the one-step set $\boldsymbol{Q}^{[s i]}$}

In robust linear control literature [16], the set $Q^{[s i]}(\Omega)$ is a polytope because there appears a single summation. However, the one-step set (13) is defined by homogeneous degree-2 polynomial inequalities in terms of the membership functions $\left(\mu_{i}\right)$. As by definition $\mu_{i}$ are positive (3), checking if a given $x$ belongs to the set for any possible $\mu$ is a copositive program, similar to those well-known in LMI-based fuzzy control where the problem has been deeply studied $[22,24]$. However, the solution to the copositive program is only asymptotic so further conservatism arises.

There is abundant literature on LMI relaxations of double fuzzy summations transforming the matrix inequality problem $\sum \mu_{i} \mu_{j} Q_{i j}>0$ presented in [22] into sufficient LMI conditions which are independent of the memberships functions $\mu_{i}$.

For brevity, on the following, a Polya-based approach analogous to [22, Theorem 1] will be discussed, albeit the proposed reasoning can be easily adapted to other relaxations ${ }^{2}$.

\footnotetext{
${ }^{2}$ As the objective of this paper is obtaining polytopic descriptions of the invariant and contractive sets, we will consider only linear relaxations in which the double sum positiveness is implied by positiveness of a collection of linear combinations of the $Q_{i j}$; such linear relaxations are, for instance, the Polya approach in [22], the triangulation approach in [24], and the non-assymptotically exact Tuan-Apkarian approach [25].
} 
As a first step, because of positiveness of $\mu_{i}$, a trivial sufficient condition for checking the negativity of $\sum_{i, j} \mu_{i} \mu_{j} Q_{i j}$ as required in (13) can be given by ensuring that all $Q_{i j}$ are negative or zero. Then, a polytopic subset, to be denoted as $\tilde{Q}_{1}(\Omega), \tilde{Q}_{1}(\Omega) \subset Q^{[s i]}(\Omega)$, can be defined by the following set of linear inequalities:

$$
\tilde{Q}_{1}(\Omega)=\left\{x \in \mathbb{R}^{n} \mid R\left(A_{i}-B_{i} F_{j}\right) x+s \leq 0, i, j=1 \ldots r\right\}
$$

However, the above result is usually very conservative (see, for instance Example 1 in section 5). Let us now discuss a methodology to overcome such conservativeness.

Notation. For brevity, let us define $\Xi$ as:

$$
\Xi=\sum_{i=1}^{r} \sum_{j=1}^{r} \mu_{i} \mu_{j} Q_{i j}(x)
$$

so the 1-step set is defined by $Q^{[s i]}=\{x \mid \Xi \leq 0\}$. For the rest of the article, the following multi-index notation from [22] is used ${ }^{3}$ :

- Boldface symbol $\mathbf{i}$ will denote a multi-index in a $d$-dimensional index set $\mathbb{I}_{d}\left(\mathbb{I}_{d}\right.$ has $r^{d}$ elements),

$$
\mathbb{I}_{d}=\left\{\mathbf{i}=\left(i_{1} i_{2} \ldots i_{d}\right) \in \mathbb{N}^{d} \mid 1 \leq i_{j} \leq r, j=1 \ldots d\right\}
$$

Also, a multidimensional summation notation will be defined as

$$
\sum_{\mathbf{i} \in \mathbb{I}_{d}} Q_{\mathbf{i}}=\sum_{i_{1}=1}^{r} \sum_{i_{2}=1}^{r} \cdots \sum_{i_{d}=1}^{r} Q_{i_{1} i_{2} \ldots i_{d}}
$$

- The product of membership functions will be denoted as:

$$
\mu_{\mathbf{i}}=\prod_{l=1}^{d} \mu_{i_{l}}=\mu_{i_{1}} \mu_{i_{2}} \ldots \mu_{i_{d}} \quad \mathbf{i} \in \mathbb{I}_{d}
$$

so, for instance, (15) can be expressed with notation (17) as $\Xi=\sum_{\mathbf{i} \in \mathbb{I}_{2}} \mu_{\mathbf{i}} Q_{\mathbf{i}}$.

- The set of unique permutations of the multi-index $\mathbf{i} \in \mathbb{I}_{d}$ is denoted by $\mathcal{P}(\mathbf{i})$, and the cardinal of $\mathcal{P}(\mathbf{i})$ is denoted as $n_{\mathbf{i}}$. This cardinal can be obtained with well-known repeated permutation formulae as $d ! / \Pi_{k=1}^{r}\left(n_{k} !\right)$, where $n_{k}$ is the number of times the integer $k$ appears in $\mathbf{i}$. For instance, for $\mathbf{i}=(3,3,1,1,1)$, $n_{\mathbf{i}}=5 ! /(2 ! 3 !)=10$ and $\mathcal{P}(\mathbf{i})=\{(3,3,1,1,1),(3,1,3,1,1),(3,1,1,3,1),(1,3,1,3,1), \ldots\}$.

The permutations will be used to group elements in multiple fuzzy summations which share the same product of membership functions: It is an evident fact that $\mathbf{j} \in \mathcal{P}(\mathbf{i}) \Rightarrow \mu_{\mathbf{j}}=\mu_{\mathbf{i}}$. For instance, $\mu_{(1,1,3,4)}=\mu_{1}^{2} \mu_{3} \mu_{4}=\mu_{(3,1,4,1)}=\mu_{(4,1,1,3)}=\ldots$.

- The subset of ordered indexes of $\mathbb{I}_{d}$ will also appear in later developments, defined as:

$$
\mathbb{I}_{d}^{+}=\left\{\mathbf{i} \in \mathbb{I}_{d} \mid i_{p} \leq i_{p+1}, \quad p=1 \ldots d-1\right\}
$$

Note that $\sum_{\mathbf{i} \in \mathbb{I}_{d}} \mu_{\mathbf{i}}=\sum_{\mathbf{i} \in \mathbb{I}_{d}^{+}} n_{\mathbf{i}} \mu_{\mathbf{i}}=1$ and for any given index $\mathbf{j} \in \mathbb{I}_{d}$, there exists a unique permutation of it, say $\mathbf{i}$, which belongs to $\mathbb{I}_{d}^{+}\left(i . e, \mathcal{P}(\mathbf{j}) \cap \mathbb{I}_{d}^{+}\right.$has only one element).

\footnotetext{
${ }^{3}$ Note that in [22] $Q_{i j}$ denoted matrices to be set up in LMI software; in this work $Q_{i j}$ are vectors to be handled by linear algebra routines, but summation notation is kept unchanged.
} 
Relaxed 1-step set. After these definitions, we take back again the problem of checking negativity of $\Xi$ in (15). As this is a double fuzzy summation which may be expanded to any desired level of nested sums, it can be expressed with this new notation as

$$
\Xi=\left(\sum_{i=1}^{r} \mu_{i}\right)^{d-2} \Xi=\sum_{\mathbf{i} \in \mathbb{I}_{d}} \mu_{\mathbf{i}} Q_{i_{1} i_{2}} \leq 0
$$

where $i_{1}, i_{2}$ are the two first elements of the multi-dimensional index i. Following the properties of the defined sets it follows that

$$
\sum_{\mathbf{i} \in \mathbb{I}_{d}} \mu_{\mathbf{i}} Q_{i_{1} i_{2}}=\sum_{\mathbf{i} \in \mathbb{I}_{d}^{+}} \sum_{\mathbf{j} \in \mathcal{P}(\mathbf{i})} \mu_{\mathbf{j}} Q_{j_{1} j_{2}}=\sum_{\mathbf{i} \in \mathbb{I}_{d}^{+}} \mu_{\mathbf{i}} \sum_{\mathbf{j} \in \mathcal{P}(\mathbf{i})} Q_{j_{1} j_{2}}=\sum_{\mathbf{i} \in \mathbb{I}_{d}^{+}} \mu_{\mathbf{i}} \tilde{Q}_{\mathbf{i}}
$$

where we define ${ }^{4} \tilde{Q}_{\mathbf{i}}=\sum_{\mathbf{j} \in \mathcal{P}(\mathbf{i})} Q_{j_{1} j_{2}}$. Therefore, a sufficient condition to prove the negativity of $\Xi$ can be obtained by checking the negativity of all the terms $\tilde{Q}_{\mathbf{i}}$ at the right-hand side of (19). Hence, the following implication is defined as "complexity- $d$ Polya conditions" in literature [22]:

$$
\tilde{Q}_{\mathbf{i}}=\sum_{\mathbf{j} \in \mathcal{P}(\mathbf{i})} Q_{j_{1} j_{2}} \leq 0 \quad \forall \mathbf{i} \in \mathbb{I}_{d}^{+} \quad \Rightarrow \quad \Xi \leq 0
$$

It can be proved that the larger $d$ is, the less conservative the conditions (20) are, but the more computationally demanding the procedure is, as the number of elements of $\mathbb{I}_{d}^{+}$increases.

Based on the fact that (20) implies $\Xi \leq 0$ in (15), the "complexity- $d$ polytopic 1 -step set" $\tilde{Q}_{d}(\Omega), d \geq 2$, of the one-step set will be defined as

$$
\tilde{Q}_{d}(\Omega)=\left\{x \in \mathbb{R}^{n} \mid \sum_{\mathbf{j} \in \mathcal{P}(\mathbf{i})} Q_{j_{1} j_{2}} \leq 0 \quad \forall \mathbf{i} \in \mathbb{I}_{d}^{+}\right\}
$$

and, from this definition of $\tilde{Q}_{d}(\Omega)$, for a fixed complexity degree $d>1$, we have:

$$
\tilde{Q}_{1}(\Omega) \subseteq \tilde{Q}_{d}(\Omega) \subseteq \tilde{Q}_{d+1}(\Omega) \subseteq Q^{[s i]}(\Omega)
$$

and $\lim _{d \rightarrow \infty} \tilde{Q}_{d}(\Omega)=Q^{[s i]}(\Omega)$ (asymptotical exactness of the Polya conditions, see [22]).

For instance, given a closed-loop system with two rules, $\tilde{Q}_{2}(\Omega)$ would be defined by inequalities:

$$
Q_{11} \leq 0 \quad Q_{22} \leq 0 \quad Q_{12}+Q_{21} \leq 0
$$

Also, $\tilde{Q}_{3}(\Omega)$ would be defined by

$$
Q_{11} \leq 0, \quad Q_{22} \leq 0, \quad Q_{11}+Q_{12}+Q_{21} \leq 0, \quad Q_{22}+Q_{12}+Q_{21} \leq 0
$$

which are obviously less restrictive than those from $\tilde{Q}_{2}(\Omega)$, proving $\tilde{Q}_{2}(\Omega) \subseteq \tilde{Q}_{3}(\Omega)$, and so on.

\section{Algorithm for computation of polytopic $\lambda$-contractive sets with Polya expansions}

Note that inequalities arising from the Polya conditions are still affine in $x$ and, hence, that the one-step sets $\tilde{Q}_{d}(\Omega)$ are polytopic, as the starting region $\Omega$ is a polytope by assumption. So, choosing a complexityparameter $d$, computation of $\tilde{Q}_{d}(\Omega)$ will obtain a polytopic subset of the shape-independent one-step set $Q^{[s i]}(\Omega)$.

\footnotetext{
${ }^{4}$ Note that, obviously, there is no need of actually carrying out the summing over $\mathcal{P}(\mathbf{i})$ as literally defined next for $\tilde{Q}_{\mathbf{i}}$. Indeed, it is possible to express $\tilde{Q}_{\mathbf{i}}=\sum_{j_{1}=1}^{r} \sum_{j_{2}=1}^{r} \mathrm{~m}_{\mathbf{i}}\left(j_{1}, j_{2}\right) Q_{j_{1} j_{2}}$ where the integer $\mathrm{m}_{\mathbf{i}}\left(j_{1}, j_{2}\right)$ can be obtained from combinatorics. Indeed, if both $j_{1}$ and $j_{2}$ are not present in $\mathbf{i}$ (or two occurrences of $j_{1}$ in case $j_{1}=j_{2}$ ), then $\mathrm{m}_{\mathbf{i}}\left(j_{1}, j_{2}\right)=0$. Otherwise, forming the $(d-2)$-dimensional multiindex, denoted as $\tilde{\mathbf{i}}$ by removing $j_{1}$ and $j_{2}$ from $\mathbf{i}$ (with removal position being arbitrary), then $\mathrm{m}_{\mathbf{i}}\left(j_{1}, j_{2}\right)$ is the number of elements of $\mathcal{P}(\tilde{\mathbf{i}})$, i.e., the previously-defined cardinal $n_{\tilde{\mathbf{i}}}$. Thus, computation of $\tilde{Q}_{\mathbf{i}}$ for each $\mathbf{i}$ is straightforward. Similar ideas with a different notation appear in, for instance, [26].
} 


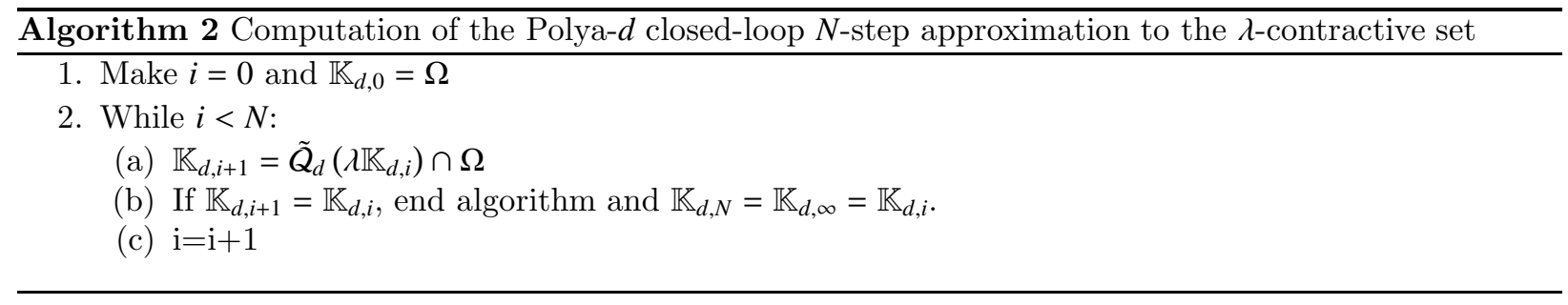

However, following the assymptotical exactness results derived from Polya argumentations [27], the set $\tilde{Q}_{d}(\Omega)$ will tend to $Q^{[s i]}(\Omega)$ as the complexity parameter $d$ tends to infinity, as stated below.

Once the choice of $d$ is made, Algorithm 2 presents a modification of Algorithm 1 using the above complexity- $d$ one-step sets. If the algorithm converges, it will allow the computation of a subset $\mathbb{K}_{d, \infty}$ of (but asymptotically equal to) the maximal shape-independent invariant or $\lambda$-contractive set $O_{\infty}^{[s i]}(\Omega)$ (i.e., contractive for all possible trajectories of (5) for arbitrary $\mu$ )

with the following properties:

- It is a polytope, because of the polytopic nature of $\tilde{Q}_{d}(\Omega)$ and $\Omega$ for any chosen $d$.

- It is $\lambda$-contractive.

- As $d$ increases the obtained converged set $\mathbb{K}_{d, \infty}$ tends to the maximal shape-independent invariant set $O_{\infty}^{[s i]}(\Omega)$ due to the assymptotical exactness, in the terms defined in the next theorem below.

Theorem 1 If $x$ belongs to the interior of the maximal shape-independent $\lambda$-contractive set in $\Omega$, denoted as $O_{\infty}^{[s i]}$, then there exist a finite $d$ such that $x$ belongs to the set $\mathbb{K}_{d, \infty}$ resulting from Algorithm 2.

Proof: Consider, for any arbitrary $h$, that we have obtained the polytopic shape-independent $h$-step set of $\Omega$, denoted as $\mathbb{K}_{h}^{[s i]}$. Then, if $x$ belongs to the interior of the $(h+1)$-step set of $\Omega$, there is a finite $d_{h}$ such that $x \in \tilde{Q}_{d}\left(\lambda \mathbb{K}_{h}^{[s i]}\right)$ due to the asymptotically exact nature of the Polya expansion [27].

Hence, as in Algorithm 1, starting from $\mathbb{K}_{0}^{[s i]}=\Omega$, an induction argumentation proves that if $x$ belongs to the interior of the $N$-step shape-independent set for $\Omega, \mathbb{K}_{N}^{[s i]}$, there is a sequence $d_{1}, \ldots, d_{N}$ such that generating $\mathbb{K}_{h+1}^{[s i]}=\tilde{Q}_{d_{h}}\left(\lambda \mathbb{K}_{h}^{[s i]}\right) \cap \Omega$, then $x \in \mathbb{K}_{N}^{[s i]}$. As conservatism of the Polya conditions decreases as $d$ increases, choosing a single complexity parameter $d=\max _{h} d_{h}$, generating now the actual sequence defined in Algorithm 2 results in $\mathbb{K}_{d, N} \supset \mathbb{K}_{N}^{[s i]}$ so that $x \in \mathbb{K}_{d, N}$.

Note: Theorem 1 asymptotically obtains the maximal shape-independent contractive set. The remaining sources of conservativeness are:

- The asymptotical nature of the theorem, so that the size of the resulting sets will depend on the user-defined parameter $d$ (chosen according to the available computational resources) and problem conditioning [27].

- The shape-independence assumption, i.e., the evaluation of the algoritm using the set in (13) instead of (11), which cannot be easily computed in a general case ${ }^{5}$.

\footnotetext{
${ }^{5}$ In some particular cases, there is some knowledge of the bounds on, say, the products of the memberships (for a particular nonlinear system we might have $\left.\mu_{2}(x) \mu_{3}(x)-0.1 \leq 0 \forall x \in \Omega\right)$ or, in general, knowing that some polynomial in the memberships fulfills $p(\mu) \leq 0$. Such kind of shape-dependent knowledge can be embedded into stability conditions via a KKT multiplier [23] and, also, into the 1-step set computation, changing $\sum_{i} \sum_{j} Q_{i j} \leq 0$ for conditions into an augmented space ( $\left.x, L\right)$ stated as $L \geq 0$, $\sum_{i} \sum_{j} Q_{i j}+L p(\mu) \leq 0$, where $L$ is the multiplier. However, these shape-dependent issues are out of the scope of the presented work, so details are omitted for brevity.
} 


\section{Relation with polytopic invariant sets for polytopic linear difference inclusions}

The presented results as mentioned at the beginning of Section 3 are clearly connected to results for polytopic LDI, where the uncertainties are bounded by a polytope. As discussed in the introduction, in previous literature results, as the parameters are unknown, there is no possibility of controlling the system by a PDC controller, contrary to TS fuzzy systems. On the other hand, when linear controllers are used, well known results are available.

Note that in the case studied in this paper, i.e. the closed-loop TS fuzzy system controlled by a PDC, can be consertively embedded in an LDI following the related literature as:

$$
x_{k+1}=G(w) x_{k} \quad \text { where } G(w) \in \operatorname{conv}\left\{\left(A_{i}-B_{i} F_{j}\right) i=1 \ldots r j=1 \ldots r\right\}
$$

If the one step set for this system is computed, the obtained set is exactly the same presented in (14) $\tilde{Q}_{1}(\Omega)$, i..e, it is a conservative estimation (subset) with respect to any other $Q_{d}$ for $d>1$.

Despite that, this approach is also interesting if we take into account that (5) can be multiplied by $\left(\sum_{i=1}^{r} \mu_{i}\right)^{d-2}$. Then, an equivalent expanded model can be presented as

$$
x_{k+1}=\sum_{\mathbf{i} \in \mathbb{I}_{d}} \mu_{\mathbf{i}} G_{\mathbf{i}} x_{k} \quad \text { where } G_{\mathbf{i}}=G_{i_{1} i_{2}}=A_{i_{1}}-B_{i_{1}} F_{i_{2}}
$$

As in Section 3.1, we can group all the coefficients $G_{i_{1} i_{2}}$ multiplied by the same value of $\mu_{\mathbf{i}}$. Then, this vector of polynomials can be expressed with the minimum number of coefficients as

$$
x_{k+1}=\sum_{\mathbf{i} \in \mathbb{I}_{d}^{+}} n_{\mathbf{i}} \mu_{\mathbf{i}} \tilde{G}_{\mathbf{i}} x_{k}
$$

Where

$$
\tilde{G}_{\mathbf{i}}=\sum_{\mathbf{j} \in \mathcal{P}(\mathbf{i})} \frac{1}{n_{\mathbf{i}}}\left(A_{j_{1}}-B_{j_{1}} F_{j_{2}}\right)
$$

As all $\mu_{\mathbf{i}}$ are positive and $\sum_{\mathbf{i} \in \mathbb{I}_{d}^{+}} n_{\mathbf{i}} \mu_{\mathbf{i}}=1$, we can define a new LDI such that

$$
x_{k+1}=G(w) x_{k} \quad \text { where } G(w) \in \operatorname{conv}\left\{\tilde{G}_{\mathbf{i}}, \forall \mathbf{i} \in \mathbb{I}_{d}^{+}\right\}
$$

As expected, as $\Omega$ is a polytope, if we compute the one step set for this system, the set $\tilde{Q}_{d}(\Omega)$ presented in Section 3.1 is found. Hence, the invariant and contractive sets from (26) inspire the developments in this work in the search for an asymptotically exact solution to the fuzzy invariant set problem.

Example 1 Let us consider the system $x_{k+1}=\mu_{1}\left(0.75 x_{k}+0.25 u_{k}\right)+\mu_{2}\left(0.75 x_{k}-0.25 u_{k}\right)$ subject to the control law $u_{k}=\mu_{1}\left(-2 x_{k}\right)+\mu_{2}\left(2 x_{k}\right)$ has the closed-loop:

$$
x_{k+1}=\sum_{i=1}^{r} \sum_{j=1}^{r} \mu_{i}\left(x_{k}\right) \mu_{j}\left(x_{k}\right)\left(A_{i}-B_{i} F_{j}\right) x_{k}=\sum_{i=1}^{r} \sum_{j=1}^{r} \mu_{i}\left(x_{k}\right) \mu_{j}\left(x_{k}\right) G_{i j} x_{k}
$$

The closed-loop system has four "vertex models" $x_{k+1}=G_{i j}\left(x_{k}\right):\left\{G_{11}: 0.25 x_{k}, G_{12}: 1.25 x_{k}, G_{21}: 1.25 x_{k}\right.$, $\left.G_{22}: 0.25 x_{k}\right\}$. Hence, considering independently the 4 vertex models as in $(22)$ would not guarantee a stable closed loop, as $G_{12}$ and $G_{21}$ are unstable: no set apart from the origin can be proved $\lambda$-contractive for $\lambda<1.25$ by iterating $Q_{1}$ in Algorithm 2. Now, in order to find a $\lambda$-contractive set we multiply the closed-loop system by $\mu_{1}+\mu_{2}=1$, we get the system (expressed as in (26))

$$
x_{k+1}=\mu_{1}^{3}\left(0.25 x_{k}\right)+3 \mu_{1}^{2} \mu_{2}\left(0.917 x_{k}\right)+3 \mu_{1} \mu_{2}^{2}\left(0.917 x_{k}\right)+\mu_{2}^{3}\left(0.25 x_{k}\right)
$$

As all the $\tilde{G}_{\mathbf{i}}$ are now stable, a $\lambda$-contractive set can be found for $\lambda=0.917$ for $d=3$ proving stability. For $d=4$, we get also $\lambda=0.917$. For $d=5$ and $d=6$, we get $\lambda=0.85$, for $d=7$ we get $\lambda=0.82$, $d=9$ gets $\lambda=0.81, d=13$ gets $\lambda=0.78, d=27$ gets $\lambda=0.77, d=43$ gets $\lambda=0.76$ and so on. 
Note that, in this simple case, we may compute an exact solution in a straightforward way; indeed, setting $\mu_{2}=1-\mu_{1}$ the closed-loop can be written as $x_{k+1}=\left(0.25+2 \mu_{1}-2 \mu_{1}^{2}\right) x_{k}$. When $\mu_{1}$ ranges in the interval [0,1], the function $\left(0.25+2 \mu_{1}-2 \mu_{1}^{2}\right)$ ranges between 0.25 and 0.75 . So, the system is actually $\lambda$-contractive up to $\lambda=0.75$. Asymptotic exactness of Polya theorems ensure that we will approach $\lambda=0.75$ as close as desired as $d$ increases. Of course, higher-order processes with more rules cannot be approached with this simplistic setup in order to determine the (decay) contraction rate, needing standard fuzzy-control LMIs [1].

\section{Lyapunov Functions and Convergence}

Note, importantly, that Theorem 1 actually shows that the proposed Algorithm 2 will ultimately obtain a contractive set larger than any level set coming from shape-independent LMI Lyapunov function search in literature, because it will asymptotically obtain the maximal set $\mathcal{O}_{\infty}^{[s i]}$, if the value of the complexity parameter $d$ can be made large enough.

However, the theorem does not give a clue on the required value of the complexity parameter and the number of algorithm steps needed to outperform a particular algorithm. In some cases, discussed in this section, results on these issues can be obtained, as follows.

Many of the LMI conditions presented in Literature are equivalent, include the condition or can be generalized, to searching for a convex Lyapunov function such that

$$
V\left(\tilde{G}_{\mathbf{i}} x_{k}\right)-V\left(\gamma x_{k}\right)<0 \quad \forall \mathbf{i} \in \mathbb{I}_{d}^{+}
$$

where the parameters of $V$ are unknown. For all this class of conditions it can be proved that the obtained invariant set is inside the proposed in the section 3.1 for the same complexity level.

Proposition 1 If there exists a convex Lyapunov function such that (27) holds, then the largest invariant set obtained from Algorithm 2 with complexity $d$ includes any level set $V(x) \leq V_{c}$ included in $\Omega$.

Proof: As $V$ is a Lyapunov function for $(26)$, the set $V(x) \leq V_{c}$ is a $\lambda$-contractive set for this system. As the set obtained from Algorithm 2 with complexity $d$ is the maximal $\lambda$-contractive set [14], proposition holds.

Moreover, if the related LMI conditions are able to find a Lyapunov function, then Algorithm 2 converges in a finite number of steps as discussed below.

Note that, as discussed above, the proposed algorithm will ultimate outperform any $V(x)$ from a Lyapunovbased approach, but if (27) does not hold for a particular choice of $V$ and $d$, then a result on which complexity level will be actually needed cannot be stated.

Proposition 2 If the closed-loop system is such that there exists a convex Lyapunov function such that the following holds with some $\gamma$ :

$$
V\left(\tilde{G}_{\mathbf{i}} x_{k}\right)-V\left(\gamma x_{k}\right)<0 \quad \forall \mathbf{i} \in \mathbb{I}_{d}^{+}
$$

then Algorithm 2 with $\lambda>\gamma$ converges in a finite number of steps. The number of steps is bounded by $m$, defined as the minimum natural number such that

$$
\max _{x \in \Omega} V\left(\gamma^{m} x\right) \leq \min _{x \in \partial \Omega} V\left(\lambda^{m} x\right)
$$

holds.

Proof: For any $x_{0}$ in $\Omega$, considering a time instant $k$, if the trajectory of (26) fulfills $x_{j} \in \Omega$ for $j \leq k$, we have:

$$
V\left(x_{k}\right) \leq \max _{x \in \Omega} V\left(\gamma^{k} x\right)
$$


Also from (29), for $k=m$, we have $V\left(x_{m}\right) \leq \max _{x \in \Omega} V\left(\gamma^{m} x\right) \leq \min _{x \in \partial \Omega} V\left(\lambda^{m} x\right)$, so $x_{m}$ belongs to a level set of the Lyapunov function which is $\gamma$-contractive and, hence, $\lambda$-contractive as $\gamma<\lambda$. Hence, using Proposition 1, we have $\Theta=\left\{x \in \mathbb{R}^{n} \mid V(x)<\min _{x \in \partial \Omega} V\left(\lambda^{k} x\right)\right\}$ belongs to the maximal $\lambda$-contractive set $\mathbb{K}_{d, \infty}$ found by the algorithm, that is, $\Theta \subset \mathbb{K}_{d, \infty}$.

In summary, all points which do not escape $\Omega$ in $m$ steps will belong to a Lyapunov level set $\Theta$. Hence, they will never escape $\Omega$. Thus, as Algorithm 2 for polytopic LDI (26) is exact, $\mathbb{K}_{d, m}$ for (26) must have already converged and $\mathbb{K}_{d, m}=\mathbb{K}_{d, m+1}=\mathbb{K}_{d, \infty}$, i.e., the algorithm will converge in at most $m$ steps.

Note: The maximum in left-hand side of (29) will be found at a non-interior point $x \in \partial \Omega$ due to the convexity of $V$. Note also that $(a) m$ does not exist for $\gamma>\lambda$ and, $(b)$ if $\gamma=\lambda$ it will only exist if $V$ were constant in all the frontier of $\Omega$ which is an uninteresting exceptional case. Hence, this justifies the choice $\lambda>\gamma$ in the theorem.

Corollary 1 For quadratic-in-the-state Lyapunov functions $m$ above is given by:

$$
m=\frac{1}{2}\left(\frac{\log V_{\min }-\log V_{\max }}{\log \gamma-\log \lambda}\right)
$$

where $V_{\min }$ and $V_{\max }$ are, respectively, the minimum and maximum values of the Lyapunov function in the frontier of $\Omega$.

Proof: Indeed, as $V(\lambda x)=\lambda^{2} V(x)$ the above formula is easily obtained from $\max _{x \in \partial \Omega} \gamma^{2 k} V(x) \leq \min _{x \in \partial \Omega} \lambda^{2 k} V(x)$, i.e., $\gamma^{2 k} V_{\max } \leq \lambda^{2 k} V_{\min }$.

Corollary 2 If Algorithm 2 converges, then there exists a polyhedral Lyapunov function proving $\lambda$-contractiveness.

Indeed, straightforwardly expressing the result of the algorithm (a polytope) in the form $\mathbb{K}_{d, \infty}=\{x \in$ $\left.\mathbb{R}^{n} \mid H x \leq 1\right\}$ for some matrix $H$, then defining $V(x)=\max _{i}\left(H_{i} x\right)$, where $H_{i}$ is the $i$-th row of matrix $H$, we have that $V(x)=1$ is the boundary of $\mathbb{K}_{d, \infty}$. Then, $V(x)$ is the sought Lyapunov function because $\mathbb{K}_{d, \infty}$ is $\lambda$-contractive, and due to the linear (time-varying) dynamics in which the original system is embedded, the other sets level $V(x) \leq \gamma$ are $\lambda$-contractive, too, as long as they are inside the modelling region $\Omega$ (i.e., for $\gamma \leq 1$.

\subsection{Fuzzy Lyapunov functions.}

As the closed-loop equations can be expressed by

$$
x_{k+1}=\sum_{\mathbf{i} \in \mathbb{I}_{d}^{+}} n_{\mathbf{i}} \mu_{\mathbf{i}} \tilde{G}_{\mathbf{i}} x_{k}
$$

consider, for instance, proving Lyapunov stability with a candidate Lyapunov function $V=x^{T} P(x) x$. Condition $V\left(x_{k+1}\right)<\lambda^{2} V\left(x_{k}\right)$ is equivalent to the well-known Schur complement expression:

$$
\left(\begin{array}{cc}
\lambda^{2} P\left(x_{k}\right) & \sum_{\mathbf{i} \in \mathbb{I}_{d}^{+}} n_{\mathbf{i}} \mu_{\mathbf{i}} \tilde{G}_{\mathbf{i}}^{T} P\left(x_{k+1}\right) \\
P\left(x_{k+1}\right) \sum_{\mathbf{i} \in \mathbb{I}_{d}^{+}} n_{\mathbf{i}} \mu_{\mathbf{i}} \tilde{G}_{\mathbf{i}} & P\left(x_{k+1}\right)
\end{array}\right)>0
$$

Let us now consider a fuzzy Lyapunov function [10] generalised to include multiple-sum expressions by setting $P\left(x_{k}\right)=\sum_{\mathbf{i} \in \mathbb{I}_{d}^{+}} n_{\mathbf{i}} \mu_{\mathbf{i}}\left(x_{k}\right) P_{\mathbf{i}}$. Then, equation (32) would get converted into

$$
\sum_{\mathbf{i}, \mathbf{j} \in \mathbb{I}_{d}^{+}} n_{\mathbf{i}} n_{\mathbf{j}} \mu_{\mathbf{i}}\left(x_{k}\right) \mu_{\mathbf{j}}\left(x_{k+1}\right)\left(\begin{array}{cc}
\lambda^{2} P_{\mathbf{i}} & \tilde{G}_{\mathbf{i}}^{T} P_{\mathbf{j}} \\
P_{\mathbf{j}} \tilde{G}_{\mathbf{i}} & P_{\mathbf{j}}
\end{array}\right)>0
$$


In order to prove it, we could prove a sufficient condition

$$
\left(\begin{array}{cc}
\lambda^{2} P_{\mathbf{i}} & \tilde{G}_{\mathbf{i}}^{T} P_{\mathbf{j}} \\
P_{\mathbf{j}} \tilde{G}_{\mathbf{i}} & P_{\mathbf{j}}
\end{array}\right)>0 \quad \forall \mathbf{i} \in \mathbb{I}_{d}^{+} \quad \forall \mathbf{j} \in \mathbb{I}_{d}^{+}
$$

Of course, quadratic Lyapunov functions are a particular case of the above setup with $P_{\mathbf{i}}=P$, resulting in level sets $\Theta=\left\{x \mid x^{T} P x \leq 1\right\}$.

Proposition 3 If LMIs (34) are feasible, then Algorithm 2 obtains a larger shape-independent $\lambda$-contractive set.

Proof: Indeed, if LMI holds, then for all $\mathbf{i}, \mathbf{j}$ we have

$$
x_{k}^{T} \tilde{G}_{\mathbf{i}} P_{\mathbf{j}} \tilde{G}_{\mathbf{i}} x_{k}-\lambda^{2} x_{k}^{T} P_{\mathbf{i}} x_{k}<0
$$

which is equivalent to:

$$
\max _{\mathbf{j}} x_{k}^{T} \tilde{G}_{\mathbf{i}} P_{\mathbf{j}} \tilde{G}_{\mathbf{i}} x_{k}-\lambda^{2} x_{k}^{T} P_{\mathbf{i}} x_{k}<0 \forall \mathbf{i}
$$

hence we have:

$$
\max _{\mathbf{j}} x_{k}^{T} \tilde{G}_{\mathbf{i}} P_{\mathbf{j}} \tilde{G}_{\mathbf{i}} x_{k}-\lambda^{2} \max _{\mathbf{l}} x_{k}^{T} P_{\mathbf{l}} x_{k}<0 \forall \mathbf{i}
$$

so this is the Lyapunov equation for system (26) and max-of-quadratics Lyapunov function $V(x)=\max _{\mathbf{i}} x^{T} P_{\mathbf{i}} x$. Then, once we have the non-fuzzy $V(x)$ for the linear difference inclusion (26), all previous results apply, in particular that the level set of the max-of-quadratics function will be smaller than the algorithm's results and it will be found in a finite number of steps bounded by Corollary 1.

Note that (34) is LMI whereas nonconservative max-of-quadratic conditions are BMI because a set of scalars (Lagrange multipliers) is required in order to restrict the above LMI to hold only for those values of $x$ such that $x^{T} P_{\mathbf{i}} x$ is larger than the rest of indices. Details omitted for brevity, see [20, Theorem 8].

\section{Shape-independence issues.}

Let us now compare the above result to the level sets of fuzzy Lyapunov functions with LMIs (33).

In order for the comparison to be fair, we will initially only consider the level sets of fuzzy Lyapunov functions that can be proved via convex programming (i.e., LMI conditions).

Under this assumption, note that to enforce via LMIs the condition $\left\{V(x)=x^{T} \sum_{\mathbf{i} \in \mathbb{I}_{d}^{+}} n_{\mathbf{i}} \mu_{\mathbf{i}} P_{\mathbf{i}} x \leq 1\right\} \subset \Omega$ in the classical fuzzy Lyapunov approach, it is mandatory to introduce separate conditions on $P_{\mathbf{i}}$ enforcing $\left\{x^{T} P_{\mathbf{i}} x \leq 1\right\} \subset \Omega$ for all $\mathbf{i}$, because the membership shape $\mu$ cannot appear in LMIs: we are forced to assume that $\mu_{\mathbf{i}}(x)$ might be equal to one for any particular $x$.

Hence, LMIs (33) plus $\left\{x^{T} P_{\mathbf{i}} x \leq 1\right\} \subset \Omega$ will provide a shape-independent level set $\Theta_{d}=\left\{V_{\mathbf{i}} \leq 1 \forall \mathbf{i}\right\}$ which is actually the level set of the max-of-quadratics Lyapunov function. Hence, previous results apply to show that the algorithms will converge to a larger set, even for the same complexity parameter $d$.

Shape-dependent level sets. Note, however, that the shape-dependent level set of the fuzzy Lyapunov function $V(x) \leq 1$ obtained with the actual evaluation of known nonlinear membership functions will be, of course, larger than $\Theta_{d}$ (and maybe not convex); indeed, $\Theta_{d}$ is, actually, the intersection of the level sets from all possible membership functions for a given set of $P_{\mathbf{i}}$.

Notwithstanding, such larger set cannot be accounted for in LMIs, but only plotted "a posteriori" once $P_{\mathbf{i}}$ are obtained (by searching a scaling factor $V_{c}$ which keeps the set $V(x)<V_{c}$ inside $\Omega$ ). The procedure is graphical by trial-and-error most of the times in $2 \mathrm{D}$ or $3 \mathrm{D}$ plots, and quite involved for higher dimensions.

As a conclusion, this graphical ad-hoc procedure can obtain different sets than our algorithm: both resulting sets will be larger than a particular $\Theta_{d}$ for a fixed feasible solution $P_{\mathbf{i}}$ but inclusion relations cannot be stated. However, the procedure is a shape-dependent workaround involving non-convex search, hence outside of the scope of the techniques compared with in the paper (restricted to be shape-independent and convex). 


\section{Example}

Let us define a fuzzy model as (2) with:

$$
\begin{array}{ll}
A_{1}=\left(\begin{array}{cc}
18.2 & 6 \\
6 & 2.9
\end{array}\right) & B_{1}=\left(\begin{array}{c}
57.9 \\
20.6
\end{array}\right) \\
A_{2}=\left(\begin{array}{ll}
8.6 & 5.8 \\
5.8 & 3.6
\end{array}\right) & B_{2}=\left(\begin{array}{c}
45.5 \\
26.5
\end{array}\right) \\
A_{3}=\left(\begin{array}{cc}
20.7 & 6.4 \\
6.4 & 1.5
\end{array}\right) & B_{3}=\left(\begin{array}{c}
52 \\
14.5
\end{array}\right)
\end{array}
$$

controlled by a PDC in the form (4) with feedback gains:

$$
F_{1}=\left(\begin{array}{ll}
-0.3 & -0.1
\end{array}\right) \quad F_{2}=\left(\begin{array}{ll}
-0.2 & -0.15
\end{array}\right) \quad F_{3}=\left(\begin{array}{ll}
-0.4 & -0.16
\end{array}\right)
$$

obtained by LMI procedures (in particular, the proposed gains are a feasible solution of the LMI problem (3.19) and (3.20) in [28]).

The polyhedral constraint set $\Lambda$ is defined such that the states hold $\left|x_{i}\right| \leq 3$ and the control action $|u| \leq 1$. This can be expressed as in (6), using matrices:

$$
T=\left(\begin{array}{cc}
-1 & 0 \\
1 & 0 \\
0 & 1 \\
0 & -1 \\
0 & 0 \\
0 & 0
\end{array}\right) \quad S=\left(\begin{array}{c}
0 \\
0 \\
0 \\
0 \\
1 \\
-1
\end{array}\right) \quad p=\left(\begin{array}{l}
3 \\
3 \\
3 \\
3 \\
1 \\
1
\end{array}\right)
$$

and, with suitable straightforward manipulations, $R$ in (9) is readily obtained.

Now, setting $\lambda=1$, if Algorithm 2 is performed in order to compute the invariant set $\mathbb{K}_{d, \infty}$, with complexity $d=1$, i.e., using the one-step set $\tilde{Q}_{1}(\Omega)$ in $(14)$, no feasible set except the origin is obtained, due to the already discussed conservatism of the choice $\tilde{Q}_{1}(\Omega)$.

Using Algorithm 2 with $d=2,3,4,5$, the invariant sets shown in Figure 1 are found, each of them bigger as $d$ increases. For this example, no bigger invariant set was found by further increasing $d$ (up to 20).

In order to compare the polytopic invariant set $\mathbb{K}_{d, \infty}$ with Lyapunov results, a family of ellipsoidal invariant sets are computed by solving the following LMI problem:

$$
\begin{aligned}
\min \alpha \text { subject to: } & \\
P_{d} & >0 \\
\tilde{G}_{\mathbf{i}}^{T} P_{d} \tilde{G}_{\mathbf{i}}-P_{d} & <0 \\
P_{d} & <\alpha W \\
\left(\begin{array}{cc}
P_{d} & a_{i} \\
a_{i}^{T} & 1
\end{array}\right) & >0
\end{aligned}
$$

where $a_{i}$ are chosen as $a_{i}=R_{i} / s_{i}$ ( $i$-th rows of matrix $R$ and vector $s$ from (9)) in order for the ellipsoid $\Theta_{d}=\left\{x \in \mathbb{R}^{n} \mid x^{T} P_{d} x \leq 1\right\}$ being a subset of $\Omega$, and $W$ is an arbitrary matrix stretching the ellipsoid shape in particular directions (actually random for this example).

Figure 2 shows how the resulting polyhedron contains the union of all the ellipsoidal invariant sets with complexity degree $d=5$, as predicted by Proposition 1 .

Repeating the algorithms for different values of the contraction (decay) rate $\lambda$, with complexity parameter $d=5$, we have the results in Figure 3. The number of iterations until convergence is depicted in Table 1, so the algorithm is able to prove a contraction rate of at least $\lambda=0.87506$ which, of course, will be equal or better than that achieved with any Lyapunov function with $d=5$. As expected, the fastest the contraction rate is, the smaller the set in which such contractiveness holds can be proved. 


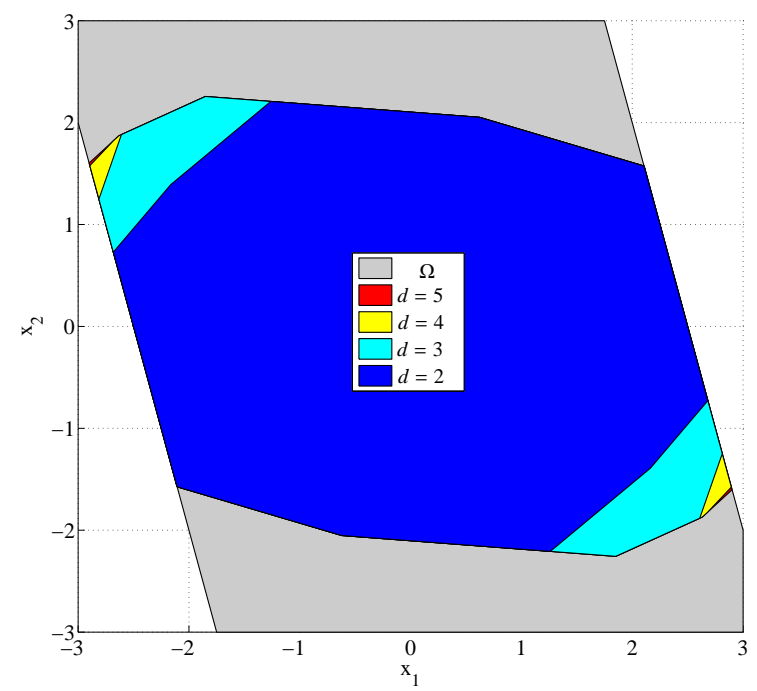

Figure 1. Valid region $\Omega$ and (suboptimal) maximal invariant sets $\mathbb{K}_{d, \infty}$, expanding with increasing $d$.

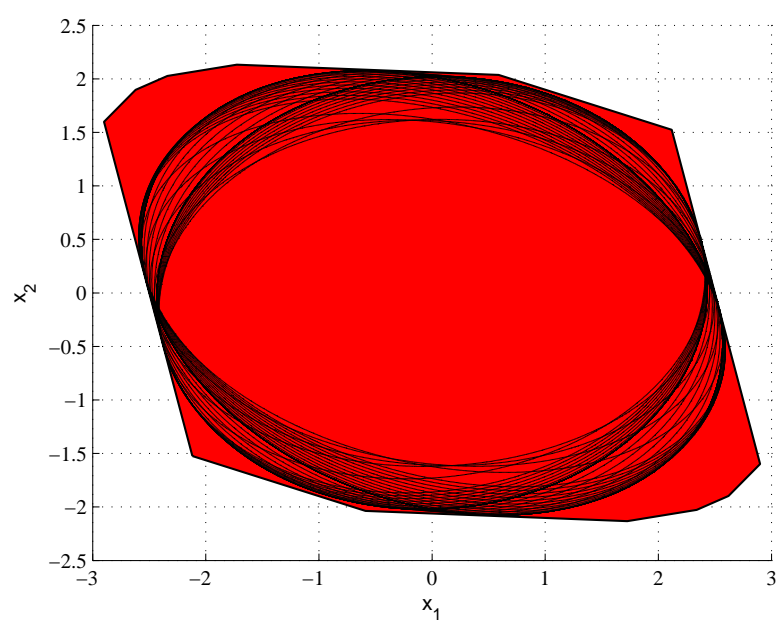

Figure 2. The resulting invariant polyhedron contains the union of all ellipsoidal invariant sets for $d=5$

Table 1. Number of iterations until convergence

\begin{tabular}{c|cccccc|c}
\hline$\lambda$ & 1 & 0.95 & 0.9 & 0.88 & 0.876 & 0.87506 & 0.87505 \\
Iters. & 4 & 4 & 4 & 8 & 12 & 25 & $>200$ \\
\hline
\end{tabular}




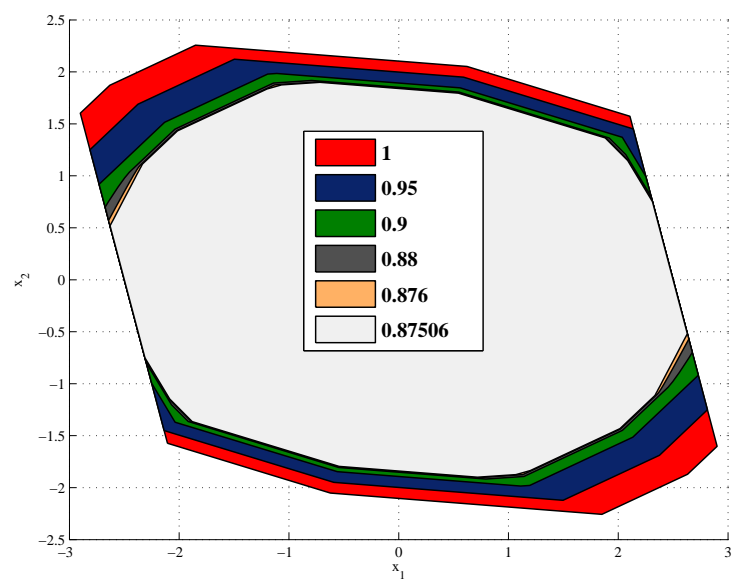

Figure 3. Maximal $\lambda$-contractive sets for different $\lambda$.

\section{Conclusions}

In this work a procedure for obtaining polytopic invariant and contractive sets for closed-loop TakagiSugeno fuzzy systems has been proposed. This procedure is based on the well-known algorithm from [14] which needs the computation of the one-step set.

In this work, no assumptions on the shape of the membership functions are made, thus the provable sets are valid for any possible membership functions (i.e., results are shape-independent). Furthermore, the invariant and $\lambda$-contractive sets obtained are polytopic if the modelling region $\Omega$ is polytopic.

The results in the work justify that the obtained invariant sets are larger than Lyapunov level sets obtained via widely used LMIs with the same linear relaxations of double-sum summations. It is also proved that, if those LMIs were feasible, the proposed algorithm would converge in a finite number of steps; also, as expected, a polyhedral Lyapunov function is obtained. An specific comparison with fuzzy Lyapunov functions is also discussed.

In fact, Theorem 1 states that the procedure would asymptotically match or outperform closed-loop stability analysis results from any conceivable Lyapunov or LDI-bounding approach if enough computing resources were available.

\section{ACKNOWLEDGEMENTS}

This work has been supported by projects DPI2011-27845-C02-01 and DPI2011-27845-C02-02, both from Spanish Government.

\section{References}

[1] K. Tanaka, T. Hori, H. O. Wang, A fuzzy Lyapunov approach to fuzzy control system design, in: Proceedings of the American Control Conference, Vol. 6, 2001, pp. 4790-4795.

[2] A. Sonbol, M. Fadali, S. Jafarzadeh, TSK fuzzy function approximators: Design and accuracy analysis, IEEE Transactions on Systems, Man, and Cybernetics, Part B: Cybernetics 42 (3) (2012) 702 -712. doi:10.1109/TSMCB.2011.2174151.

[3] K. Tanaka, T. Ikeda, H. O. Wang, Robust stabilization of a class of uncertain nonlinear systems via fuzzy control: Quadratic stabilizability, $H_{\infty}$ control theory, and linear matrix inequalities, IEEE Transactions on Fuzzy Systems 4 (1) (1996) 1-13.

[4] C. Ariño, A. Sala, Design of multiple-parameterisation PDC controllers via relaxed conditions for multi-dimensional fuzzy summations, in: IEEE International Conference on Fuzzy Systems, 2007.

[5] A. Sala, T. M. Guerra, R. Babuška, Perspectives of fuzzy systems and control, Fuzzy Sets and Systems 156 (3) (2005) $432-444$.

[6] M. Seidi, A. H. D. Markazi, Performance-oriented parallel distributed compensation, Journal of the Franklin Institute 348 (7) (2011) 1231-1244. 
[7] T. Zou, S. Li, Stabilization via extended nonquadratic boundedness for constrained nonlinear systems in Takagi-Sugeno's form, Journal of the Franklin Institute 348 (10) (2011) 2849-2862.

[8] J. Slotine, W. Li, et al., Applied nonlinear control, Vol. 199, Prentice-Hall Englewood Cliffs, NJ, 1991.

[9] F. Blanchini, Ultimate boundedness control for uncertain discrete-time systems via set-induced Lyapunov functions, in: proc. of 30th IEEE Conf. on Decision and Control, IEEE, 1991, pp. 1755-1760.

[10] T. Guerra, L. Vermeiren, LMI-based relaxed nonquadratic stabilization conditions for nonlinear systems in the TakagiSugeno's form, Automatica 40 (5) (2004) 823-829.

[11] F. Blanchini, Set invariance in control, Automatica 35 (11) (1999) 1747-1767.

[12] D. Mayne, J. Rawlings, C. Rao, P. Scokaert, Constrained model predictive control: Stability and optimality, Automatica 36 (6) (2000) 789-814.

13] M. Kvasnica, P. Grieder, M. Baotić, M. Morari, Multi-parametric toolbox (MPT), Hybrid systems: computation and control (2004) 121-124.

[14] E. Kerrigan, Robust constraint satisfaction: Invariant sets and predictive control, Ph.D. thesis, Department of Engineering, University of Cambridge, UK (2000).

[15] E. G. Gilbert, K. T. Tan, Linear systems with state and control constraints: The theory and application of maximal output admissible sets, IEEE Transactions on Automatic Control 36 (9) (1991) 1008-1020.

[16] B. Pluymers, J. A. Rossiter, J. A. K. Suykens, B. De Moor, The efficient computation of polyhedral invariant sets for linear systems with polytopic uncertainty, in: Proceedings of the American Control Conference, Vol. 2, 2005 , pp. 804-809.

[17] S. Raković, E. Kerrigan, D. Mayne, K. Kouramas, Optimized robust control invariance for linear discrete-time systems: Theoretical foundations, Automatica 43 (5) (2007) 831-841.

[18] M. Lazar, W. Heemels, S. Weiland, A. Bemporad, Stabilizing model predictive control of hybrid systems, Automatic Control, IEEE Transactions on 51 (11) (2006) 1813-1818.

[19] A. Sala, On the conservativeness of fuzzy and fuzzy-polynomial control of nonlinear systems, Annual Reviews in Control 33 (1) (2009) 48-58.

[20] T. Hu, F. Blanchini, Non-conservative matrix inequality conditions for stability/stabilizability of linear differential inclusions, Automatica 46 (1) (2010) 190-196.

[21] E. Pérez, C. Ariño, F. Blasco, M. Martínez, Maximal closed loop admissible set for linear systems with non-convex polyhedral constraints, Journal of Process Control 21 (4) (2011) 529-537.

[22] A. Sala, C. Ariño, Asymptotically necessary and sufficient conditions for stability and performance in fuzzy control: Applications of Polya's theorem, Fuzzy Sets and Systems 158 (24) (2007) 2671-2686.

[23] A. Sala, C. Ariño, Relaxed stability and performance conditions for Takagi-Sugeno fuzzy systems with knowledge on membership function overlap, Systems, Man, and Cybernetics, Part B: Cybernetics, IEEE Transactions on 37 (3) (2007) $727-732$.

[24] A. Kruszewski, A. Sala, T. Guerra, C. Ariño, A triangulation approach to asymptotically exact conditions for fuzzy summations, Fuzzy Systems, IEEE Transactions on 17 (5) (2009) 985-994.

[25] H. Tuan, P. Apkarian, T. Narikiyo, Y. Yamamoto, Parameterized linear matrix inequality techniques in fuzzy control system design, Fuzzy Systems, IEEE Transactions on 9 (2) (2001) 324-332.

[26] B. Ding, Homogeneous polynomially nonquadratic stabilization of discrete-time takagi-sugeno systems via nonparallel distributed compensation law, Fuzzy Systems, IEEE Transactions on 18 (5) (2010) 994-1000.

[27] V. Powers, B. Reznick, A new bound for Pólya's theorem with applications to polynomials positive on polyhedra, Journal of Pure and Applied Algebra 164 (1) (2001) 221-229.

[28] K. Tanaka, H. O. Wang, Fuzzy control systems design and analysis: a linear matrix inequality approach, Wiley. com, 2004. 